\title{
Looking Inside the Fascinating Nanoworld Controlling Light Emission from InGaN/GaN Quantum Well Devices
}

\author{
C.J. Humphreys, ${ }^{*}$ R.A. Oliver, ${ }^{*}$ M.J. Kappers, ${ }^{*}$ S.E. Bennett, ${ }^{*}$ D. Parris, ${ }^{* *}$ P. Dawson, ${ }^{* *}$ M.J. \\ Godfrey, ${ }^{* *}$ P.H. Clifton, ${ }^{* * *}$ D.J. Larson, ${ }^{* * *}$ R.M. Ulfig, ${ }^{* * *}$ D.W. Saxey, ${ }^{* * * *}$ A. Cerezo $* * * *$ \\ * Department of Materials Science and Metallurgy, University of Cambridge, Pembroke Street, \\ Cambridge CB2 3QZ, UK \\ ** Department of Physics and Astronomy, Photon Science Institute, The University of \\ Manchester, Manchester M13 9PL, UK \\ *** Imago Scientific Instruments, 5500 Nobel Drive, Madison, WI 53711, USA \\ **** Department of Materials, University of Oxford, Parks Road, Oxford OX1 3PH, UK
}

For the last fifteen years scientists have been puzzled over why blue, green and white GaN-based quantum-well LEDs are so bright even though the dislocation density is very high, typically $10^{9} \mathrm{~cm}^{-2}$ [1]. Initially it was believed that this was because the InGaN quantum well underwent spinodal decomposition into nanometer-size indium rich clusters during growth by MOCVD or MBE [2]. These clusters were believed to localise the carriers, thus preventing their diffusion to dislocations which would quench the light emission since they are non-radiative recombination centres. Such clusters were observed by electron microcopy [3]. However, it was shown that electron-beam damage can rapidly produce In-rich clusters in InGaN, and that such clusters are not visible in lowdose electron microscopy [4, 5, 6], although this has been disputed [7]. In addition, Atom Probe Tomography (APT) has not revealed any In-rich clusters and the In distribution in the quantum wells is consistent with InGaN being a random alloy [8]. Karpov [9] has shown that in a strained InGaN quantum well, the strain suppresses the spinodal decomposition, so no indium-rich clusters are expected thermodynamically for blue and green InGaN LEDs, in agreement with our experiments.

However, if In-rich clusters do not exist in blue and green InGaN quantum wells, what mechanism(s) is localizing the carriers? We have identified three different mechanisms, on length scales ranging from one $\mathrm{nm}$ to one hundred nm. First, our quantum mechanical modeling shows that random statistical fluctuations in the InGaN alloy localise the holes on a 1-2 nm length scale. Second, both high resolution electron microscopy [10] and APT [11] reveal that monolayer-height interface steps, typically about $5 \mathrm{~nm}$ across, exist at the upper InGaN/GaN quantum well interface. Our modeling shows that these interface steps localise the electrons on a 5-10 nm length scale. Third, we have observed gross thickness fluctuations in the InGaN quantum well width, on a lateral length scale of typically 50-100 nm, in some bright commercial LEDs as well as in some of those we have grown [12]. The majority of dislocations were observed to pass through the gaps in the wells, thus localizing the carriers away from the dislocations on a 50-100 nm length scale. All three mechanisms are strong enough to localise the carriers at room temperature. TEM and APT are therefore absolutely key techniques for solving the long-standing problem of why InGaN/GaN LEDs are so bright when the dislocation density is so high.

References

[1] C.J. Humphreys, MRS Bulletin, Special Issue : Harnessing Materials for Energy, 33 (2008) 459.

[2] I.C. Ho and G.B. Stringfellow, Appl. Phys. Lett. 69 (1996) 2701. 
[3] Y. Narukawa et al., Appl. Phys. Lett. 70 (1997) 981

[4] T.M. Smeeton et al., Phys. Status Solidi, B 240 (2003) 297

[5] J.P. O’Neill et al., Appl. Phys. Lett. 83 (2003) 1965

[6] T.M. Smeeton et al., Appl. Phys. Lett. 83 (2003) 5419

[7] T.P. Bartel et al., Phil. Mag. 87 (2007) 1983

[8] M.J. Galtrey et al., Appl. Phys. Lett. 90 (2007) 061903

[9] S.Y. Karpov, MRS Internet Journal of Nitride Semiconductor Research, 3 (1998) 16

[10] D.M. Graham et al., J. Appl. Phys. 97 (2005) 103508

[11] M.J. Galtrey et al., J. Appl. Phys. 104 (2008) 013524

[12] N.K. van der Laak et al., Appl. Phys. Lett. 90 (2007) 121911

[13] This research was supported by the Engineering and Physical Sciences Research Council

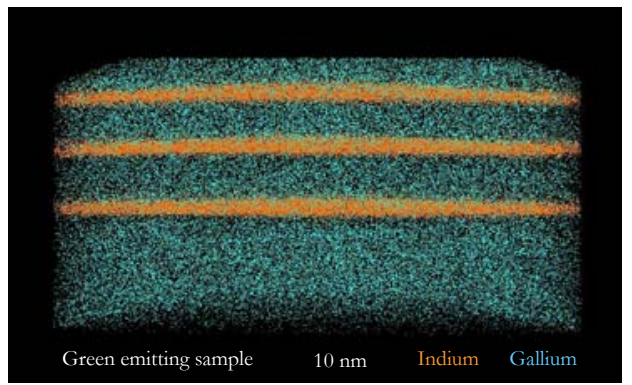

FIG. 1. Atom Probe Tomography image of three green-emitting InGaN quantum wells with GaN barriers. Each dot is one atom. Indium atoms are orange, gallium atoms are blue. Not all gallium atoms displayed (or else individual $\mathrm{Ga}$ atoms would be too dense to be visible).

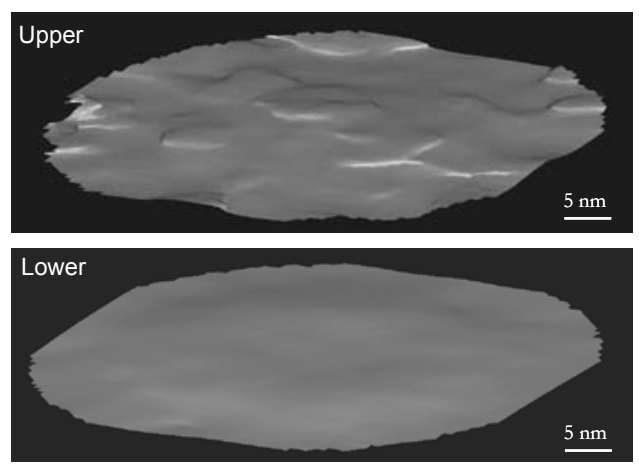

FIG. 2. Isosurfaces (surfaces on which the In concentration is constant) of InGaN/GaN quantum well interfaces from APT data. Top image is the upper interface and bottom image is the lower interface. Average r.m.s. roughness of upper interface: $0.34 \mathrm{~nm}$ and lower interface: $0.18 \mathrm{~nm}$. Monolayer-high interface steps clearly visible on upper interface.

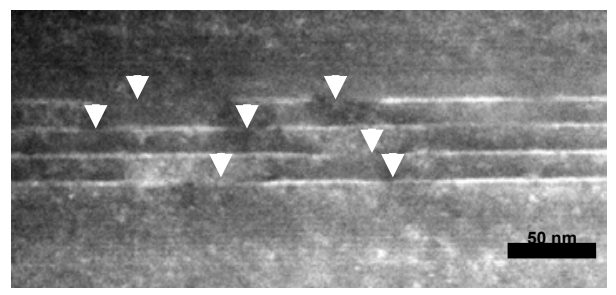

FIG. 3. TEM image of InGaN quantum wells with gross thickness fluctuations. 